\title{
Approximations of the generalized-Euler-constant function and the generalized Somos' quadratic recurrence constant
}

\section{$\operatorname{Aimin} X u^{1 *}$}

"Correspondence:

xuaimin1009@hotmail.com

${ }^{1}$ Institute of Mathematics, Zhejiang

Wanli University, Ningbo, China

\begin{abstract}
In this paper, we provide an estimate for approximating the generalized-Euler-constant function $\gamma(z)=\sum_{k=1}^{\infty} z^{k-1}\left(\frac{1}{k}-\ln \frac{k+1}{k}\right)$ by its partial sum $\gamma_{N-1}(z)$ when $0<z<1$. We obtain an asymptotic expansion for the generalized-Euler-constant function and show that the coefficients of the asymptotic expansion are explicitly expressed by the Eulerian fractions. Also, we find a recurrence relation for those coefficients. Using its relation with the generalized-Euler-constant function, we establish two inequalities for the generalized Somos' quadratic recurrence constant. Moreover, two asymptotic expansions for the natural logarithm of the generalized Somos quadratic recurrence constant are presented.
\end{abstract}

MSC: $11 Y 60 ; 33 E 20 ; 41 A 60 ; 26 D 15$

Keywords: Asymptotic expansion; Eulerian fraction; Generalized-Euler-constant function; Inequality; Somos' quadratic recurrence constant

\section{Introduction}

Somos' quadratic recurrence constant $\sigma$ arises in the study of the asymptotic behavior of the sequence (see $[8$, p. 446] and $[21,27])$ :

$$
\begin{aligned}
g_{n} \sim & \frac{\sigma^{2^{n}}}{n}\left(1+\frac{2}{n}-\frac{1}{n^{2}}+\frac{4}{n^{3}}-\frac{21}{n^{4}}+\frac{138}{n^{5}}-\frac{1091}{n^{6}}+\frac{10,088}{n^{7}}-\frac{106,918}{n^{8}}\right. \\
& \left.+\frac{1,279,220}{n^{9}}-\frac{17,070,418}{n^{10}}+\frac{251,560,472}{n^{11}}-\frac{4,059,954,946}{n^{12}}+\cdots\right)^{-1},
\end{aligned}
$$

where $g_{n}$ are recursively defined by

$$
g_{0}=1, \quad g_{n}=n g_{n-1}^{2}, \quad n=1,2, \ldots,
$$

with the first few terms

$$
g_{0}=1, \quad g_{1}=1, \quad g_{2}=2, \quad g_{3}=12, \quad g_{4}=576, \quad g_{5}=1,658,880, \ldots
$$

(c) The Author(s) 2019. This article is distributed under the terms of the Creative Commons Attribution 4.0 International License (http://creativecommons.org/licenses/by/4.0/), which permits unrestricted use, distribution, and reproduction in any medium, provided you give appropriate credit to the original author(s) and the source, provide a link to the Creative Commons license, and indicate if changes were made. 
The constant $\sigma$ is usually defined by

$$
\sigma=\sqrt{1 \sqrt{2 \sqrt{3 \sqrt{4 \cdots}}}}=\prod_{k=1}^{\infty} k^{\frac{1}{2^{k}}}=1.66168794 \cdots
$$

or

$$
\sigma=\exp \left\{-\int_{0}^{1} \frac{1-x}{(2-x) \ln x} d x\right\}=\exp \left\{-\int_{0}^{1} \int_{0}^{1} \frac{x}{(2-x y) \ln (x y)} d x d y\right\}
$$

See [9, 20-22]. The constant $\sigma$ appears in many important problems in pure and applied analysis and it has been investigated by a large number of researchers $[3,5,9,10,12,13$, $15,17-19,25,30]$.

Recently, Sondow and Hadjicostas [25] introduced and studied the generalized-Eulerconstant function $\gamma(z)$ defined by the power series

$$
\gamma(z)=\sum_{k=1}^{\infty} z^{k-1}\left(\frac{1}{k}-\ln \frac{k+1}{k}\right)
$$

when $|z| \leq 1$. There exist integral representations for the function

$$
\gamma(z)=\int_{0}^{1} \frac{1-x+\ln x}{(1-x z) \ln x} d x=-\int_{0}^{1} \int_{0}^{1} \frac{1-x}{(1-x y z) \ln (x y)} d x d y .
$$

Its values include Euler's constant $\gamma=\gamma(1)$ and the "alternating Euler constant" $\log \frac{4}{\pi}=$ $\gamma(-1)$, see for example $[23,24]$. In particular, at $z=1 / 2$, the function takes the value

$$
\gamma\left(\frac{1}{2}\right)=2 \ln \frac{2}{\sigma}
$$

which is equivalent to

$$
\sigma=2 \exp \left\{-\frac{1}{2} \gamma\left(\frac{1}{2}\right)\right\}
$$

Mortici [15] proved that, for $n \geq 1$, it follows that

$$
\frac{270(n+1)}{2^{n}\left(270 n^{3}+1530 n^{2}+1065 n+6293\right)}<\gamma\left(\frac{1}{2}\right)-\gamma_{n}\left(\frac{1}{2}\right)<\frac{18}{2^{n}\left(18 n^{2}+84 n-13\right)},
$$

where the partial sum of $\gamma(z)$

$$
\gamma_{n}(z)=\sum_{k=1}^{n} z^{k-1}\left(\frac{1}{k}-\ln \frac{k+1}{k}\right), \quad|z| \leq 1
$$

Lu and Song [13] improved Mortici's estimate and proved that, for $n \geq 1$,

$$
\frac{690 n^{2}+3524 n+145}{6\left(2^{n}\right)(n+1)^{2}\left(115 n^{2}+894 n+779\right)}<\gamma\left(\frac{1}{2}\right)-\gamma_{n}\left(\frac{1}{2}\right)
$$




$$
<\frac{48 n+127}{3\left(2^{n}\right)(16 n+85)(n+1)^{2}} .
$$

You and Chen [30] improved these inequalities by using continued fraction. Very recently, Chen and Han [5] obtained new lower bounds for $\gamma(1 / 2)-\gamma_{n}(1 / 2)$ :

$$
\begin{aligned}
\frac{1}{2^{n}} & \left(\frac{1}{(n+1)^{2}}-\frac{8}{3(n+1)^{3}}+\frac{23}{2(n+1)^{4}}-\frac{332}{5(n+1)^{5}}+\frac{479}{(n+1)^{6}}-\frac{29,024}{7(n+1)^{7}}\right) \\
& <\gamma\left(\frac{1}{2}\right)-\gamma_{n}\left(\frac{1}{2}\right) \\
& <\frac{1}{2^{n}}\left(\frac{1}{(n+1)^{2}}-\frac{8}{3(n+1)^{3}}+\frac{23}{2(n+1)^{4}}-\frac{332}{5(n+1)^{5}}+\frac{479}{(n+1)^{6}}\right) .
\end{aligned}
$$

In their paper, Chen and Han pointed out that the lower bound in (1.11) is sharper than the one in (1.10) for $n \geq 24$, and the upper bound in (1.11) is sharper than the one in (1.10) for $n \geq 18$. Moreover, they gave the following asymptotic expansion:

$$
\begin{aligned}
& \gamma\left(\frac{1}{2}\right)-\gamma_{n}\left(\frac{1}{2}\right) \\
& \quad \sim \frac{1}{2^{n}}\left\{\frac{a_{2}}{(n+1)^{2}}+\frac{a_{3}}{(n+1)^{3}}+\frac{a_{4}}{(n+1)^{4}}+\frac{a_{5}}{(n+1)^{5}}+\frac{a_{6}}{(n+1)^{6}}+\cdots\right\}, \quad n \rightarrow \infty
\end{aligned}
$$

with a recursive formula for successively determining the coefficients

$$
\begin{aligned}
& a_{2}=1 \\
& a_{k}=\frac{2(-1)^{k}}{k}+\sum_{j=2}^{k-1}(-1)^{k-j}\left(\begin{array}{c}
k-1 \\
k-j
\end{array}\right) a_{j}, \quad k \geq 3 .
\end{aligned}
$$

Recently, there have been several interesting works related to approximations of Euler's constant $\gamma=\gamma(1)$, see for example $[4,16,28]$ and the references therein. Also, some works related to approximations of $\gamma(z)$ at special values $1 / 2,1 / 3$, and $1 / 4$ appear in $[3,5$, $13-15,29,30]$. Motivated by these, the first aim of this paper is to give an approximation for $\gamma(z)$ when $0<z<1$. Specifically, we give a general inequality for the error bound of $\gamma(z)-\gamma_{N-1}(z)$. The second aim is to establish an asymptotic expansion for $\gamma(z)-\gamma_{N-1}(z)$ whose coefficients can be computed explicitly and recursively. In particular, we generalize inequality (1.11) and asymptotic expansion (1.12) due to Chen and Han. Using the relation between the generalized Somos' quadratic recurrence constant and the generalizedEuler-constant function established by Sondow and Hadjicostas [25], we find approximate estimates for the generalized Somos' quadratic recurrence constant and its natural logarithm, respectively. Moreover, two asymptotic expansions for the natural logarithm of the generalized Somos' quadratic recurrence constant are presented.

\section{Preliminaries}

Let us recall the following classical result $[2,7,11]$ :

$$
\sum_{k=0}^{\infty} k^{n} z^{k}=\frac{A_{n}(z)}{(1-z)^{n+1}}, \quad|z|<1,
$$


where $A_{n}(z)$ is the Eulerian polynomial of degree $n$, and it can be defined by the exponential generating function

$$
\sum_{n=0}^{\infty} A_{n}(z) \frac{t^{n}}{n !}=\frac{1-z}{1-z e^{t(1-z)}} .
$$

By (2.2), $A_{n}(x)$ may be explicitly written as

$$
\begin{aligned}
& A_{0}(z)=1, \\
& A_{n}(z)=\sum_{k=1}^{n} A(n, k) z^{k}, \quad n \geq 1,
\end{aligned}
$$

where $A(n, k)$ are known as the Eulerian numbers, the numbers of permutations of the numbers 1 to $n$ in which exactly $k$ elements are greater than the previous element, and they can be expressed by

$$
A(n, k)=\sum_{i=1}^{k}(-1)^{i}\left(\begin{array}{c}
n+1 \\
i
\end{array}\right)(k-i)^{n} .
$$

Therefore, one may easily obtain

$$
A_{0}(z)=1, \quad A_{1}(z)=z, \quad A_{2}(z)=z^{2}+z, \quad A_{3}(z)=z^{3}+4 z^{2}+z, \ldots
$$

For $z \neq 1$, denote the Eulerian fraction

$$
b_{n}(z)=\frac{A_{n}(z)}{(1-z)^{n+1}} .
$$

From (2.2) it is clear that

$$
\sum_{k=0}^{\infty} b_{k}(z) \frac{t^{k}}{k !}=\frac{1}{1-z e^{t}},
$$

which implies that $b_{k}(z)$ can be computed by the following recurrence relation:

$$
\begin{aligned}
& b_{0}(z)=\frac{1}{1-z}, \\
& b_{k}(z)=\frac{z}{1-z} \sum_{j=0}^{k-1}\left(\begin{array}{l}
k \\
j
\end{array}\right) b_{j}(z), \quad k \geq 1 .
\end{aligned}
$$

Also, we can obtain an explicit expression [11] as follows:

$$
b_{k}(z)=\sum_{j=0}^{k} j ! S(k, j) \frac{z^{j}}{(1-z)^{j+1}},
$$

where $S(k, j)$ are the Stirling numbers of the second kind.

It is worth noting that a general summation formula for power series using the Eulerian fractions was established in the recent paper [26]. 
Lemma 2.1 (cf. Theorem 4.2 [26]) Let $m$ be a positive integer, and both $P$ and $Q$ be nonnegative integers. Suppose that $f(x)$ has the $(m+1)$ th continuous derivative on $[P, Q]$. For $0<z<1, f^{(m)}(x)$ and $f^{(m+1)}(x)$ keep opposite signs in $(P, Q)$. Then there exists a number $\theta \in(0,1)$ such that

$$
\sum_{k=P}^{Q-1} f(k) z^{k}=\sum_{k=0}^{m-1} \frac{b_{k}(z)}{k !}\left[z^{P} f^{(k)}(P)-z^{Q} f^{(k)}(Q)\right]+\theta \frac{b_{m}(z)}{m !}\left[z^{P} f^{(m)}(P)-z^{Q} f^{(m)}(Q)\right] .
$$

Remark 2.1 It is well known that the Euler-Maclaurin formula [1] is usually applied to treat the summation $S=\sum_{k=a}^{b} f(k)$, and it was also used to approximate the generalizedEuler-constant function $\gamma(z)$, see [12]. It seems that the above lemma is more suitable to treat a general summation formula for power series with the form $S=\sum_{k=a}^{b} f(k) z^{k}(0<z<$ 1). The lemma provides us a new approach to estimate $\gamma(z)$, and it will play a key role in the next section.

Remark 2.2 In particular, if

$$
\lim _{Q \rightarrow+\infty} z^{Q} f^{(k)}(Q)=0, \quad 0 \leq k \leq m
$$

as $Q \rightarrow+\infty$, then (2.10) can be rewritten as

$$
\sum_{k=P}^{\infty} f(k) z^{k}=z^{P}\left\{\sum_{k=0}^{m-1} \frac{b_{k}(z)}{k !} f^{(k)}(P)+\theta \frac{b_{m}(z)}{m !} f^{(m)}(P)\right\} .
$$

Obviously, taking $f(x)=x^{n}, P=0$, and $m=n+1,(2.12)$ reduces to $(2.1)$.

\section{Estimate of the generalized-Euler-constant function}

Let $N$ be a positive integer. In this section, by using (2.12) we first give a general inequality for the error bound of $\gamma(z)-\gamma_{N-1}(z)$ when $0<z<1$.

Theorem 3.1 Let $p$ and $q$ be any positive integers. If $N \geq-\frac{c_{2 p+3}(z)}{c_{2 p+2}(z)}$, then we have

$$
\gamma(z)-\gamma_{N-1}(z)>z^{N-1} \sum_{k=2}^{2 p+1} \frac{c_{k}(z)}{N^{k}}, \quad 0<z<1
$$

If $N \geq-\frac{c_{2 q+2}(z)}{c_{2 q+1}(z)}$, then we have

$$
\gamma(z)-\gamma_{N-1}(z)<z^{N-1} \sum_{k=2}^{2 q} \frac{c_{k}(z)}{N^{k}}, \quad 0<z<1
$$

In the above inequalities, the coefficients $c_{k}(z)$ are explicitly expressed by

$$
c_{k}(z)=\frac{(-1)^{k}}{k}\left[\frac{1-z}{z} b_{k}(z)-k b_{k-1}(z)\right], \quad k \geq 2
$$

where $b_{k}(z)$ are the Eulerian fractions introduced in the second section. 
Proof Write

$$
f(t)=\frac{1}{t}-\ln \frac{t+1}{t} .
$$

After simple calculations, we arrive at

$$
\begin{aligned}
f^{(i)}(t) & =(-1)^{i}(i-1) !\left[\frac{i}{t^{i+1}}+\frac{1}{(t+1)^{i}}-\frac{1}{t^{i}}\right] \\
& =\frac{(-1)^{i}(i-1) !}{t^{i+1}(t+1)^{i}} \sum_{k=0}^{i}\left(\begin{array}{l}
i \\
k
\end{array}\right)\left(i-\frac{k}{i-k+1}\right) t^{k}, \quad i \geq 1,
\end{aligned}
$$

which implies that

$$
(-1)^{i} f^{(i)}(t)>0, \quad t>0 .
$$

By (3.4) and (3.6), for each $i \geq 0$, we also have

$$
\lim _{t \rightarrow+\infty} z^{t} f^{(i)}(t)=0, \quad 0<z<1
$$

Therefore, according to (2.12), for $0<z<1$, there exists $\theta \in(0,1)$ such that

$$
\begin{aligned}
\gamma(z)-\gamma_{N-1}(z) & =\frac{1}{z} \sum_{k=N}^{\infty}\left(\frac{1}{k}-\ln \frac{k+1}{k}\right) z^{k} \\
& =z^{N-1}\left[\sum_{k=0}^{m-1} \frac{b_{k}(z)}{k !} f^{(k)}(N)+\theta \frac{b_{m}(z)}{m !} f^{(m)}(N)\right],
\end{aligned}
$$

where $m$ is a positive integer. It follows from (3.4) and (3.5) that

$$
\begin{aligned}
\gamma(z)-\gamma_{N-1}(z)= & z^{N-1}\left\{b_{0}(z)\left(\frac{1}{N}-\ln \frac{N+1}{N}\right)\right. \\
& +\sum_{k=1}^{m-1} \frac{(-1)^{k} b_{k}(z)}{k}\left(\frac{k}{N^{k+1}}+\frac{1}{(N+1)^{k}}-\frac{1}{N^{k}}\right) \\
& \left.+\theta \frac{(-1)^{m} b_{m}(z)}{m}\left(\frac{m}{N^{m+1}}+\frac{1}{(N+1)^{m}}-\frac{1}{N^{m}}\right)\right\} .
\end{aligned}
$$

By the Taylor expansion we have

$$
\begin{aligned}
& \ln \frac{N+1}{N}=\sum_{j=1}^{m} \frac{(-1)^{j-1}}{j N^{j}}+\frac{(-1)^{m}}{(m+1) N^{m+1}} \frac{1}{\left(1+\frac{\phi_{0}}{N}\right)^{m+1}}, \\
& \frac{1}{(N+1)^{k}}=\frac{1}{N^{k}}\left\{\sum_{j=0}^{m-k}\left(\begin{array}{c}
k+j-1 \\
j
\end{array}\right) \frac{(-1)^{j}}{N^{j}}+\left(\begin{array}{c}
m \\
k-1
\end{array}\right) \frac{(-1)^{m+1-k}}{N^{m+1-k}\left(1+\frac{\phi_{k}}{N}\right)^{m+1}}\right\},
\end{aligned}
$$


where $0<\phi_{k}<1$ for $k=0,1, \ldots, m-1$. When $m \geq 3$, substituting (3.11) and (3.12) into (3.10) yields

$$
\begin{aligned}
\gamma(z)-\gamma_{N-1}(z)= & z^{N-1}\left\{\sum_{k=2}^{m} \frac{(-1)^{k} b_{0}(z)}{k N^{k}}+\frac{(-1)^{m+1} b_{0}(z)}{(m+1) N^{m+1}} \frac{1}{\left(1+\frac{\phi_{0}}{N}\right)^{m+1}}\right. \\
& +\sum_{k=2}^{m} \frac{(-1)^{k-1} b_{k-1}(z)}{N^{k}}+\sum_{k=1}^{m-1} \sum_{j=0}^{m-k} \frac{(-1)^{k+j} b_{k}(z)}{k N^{k+j}}\left(\begin{array}{c}
k+j-1 \\
j
\end{array}\right) \\
& \left.+\frac{(-1)^{m+1}}{N^{m+1}} \sum_{k=1}^{m-1} \frac{b_{k}(z)}{k} \frac{\left(\begin{array}{c}
m \\
k-1
\end{array}\right)}{\left(1+\frac{\phi_{k}}{N}\right)^{m+1}}-\sum_{k=1}^{m-1} \frac{(-1)^{k} b_{k}(z)}{k} \frac{1}{N^{k}}+\epsilon\right\},
\end{aligned}
$$

where

$$
\epsilon=\theta \frac{(-1)^{m} b_{m}(z)}{m}\left(\frac{m}{N^{m+1}}+\frac{1}{(N+1)^{m}}-\frac{1}{N^{m}}\right) .
$$

Taking $u=k+j$, we obtain

$$
\begin{aligned}
& \sum_{k=1}^{m-1} \sum_{j=0}^{m-k} \frac{(-1)^{k+j} b_{k}(z)}{k N^{k+j}}\left(\begin{array}{c}
k+j-1 \\
j
\end{array}\right) \\
& \quad=\sum_{k=1}^{m-1} \frac{(-1)^{k} b_{k}(z)}{k N^{k}}+\sum_{k=1}^{m-1} \sum_{j=1}^{m-k} \frac{(-1)^{k+j} b_{k}(z)}{k N^{k+j}}\left(\begin{array}{c}
k+j-1 \\
j
\end{array}\right) \\
& =\sum_{k=1}^{m-1} \frac{(-1)^{k} b_{k}(z)}{k N^{k}}+\sum_{u=2}^{m} \frac{(-1)^{u}}{N^{u}} \sum_{k=1}^{u-1} \frac{b_{k}(z)}{k}\left(\begin{array}{c}
u-1 \\
k-1
\end{array}\right) \\
& =\sum_{k=1}^{m-1} \frac{(-1)^{k} b_{k}(z)}{k N^{k}}+\sum_{u=2}^{m-1} \frac{(-1)^{u}}{N^{u}} \sum_{k=1}^{u-1} \frac{b_{k}(z)}{k}\left(\begin{array}{c}
u-1 \\
k-1
\end{array}\right)+\frac{(-1)^{m}}{N^{m}} \sum_{k=1}^{m-1}\left(\begin{array}{c}
m-1 \\
k-1
\end{array}\right) \frac{b_{k}(z)}{k} .
\end{aligned}
$$

Since

$$
\frac{1}{k}\left(\begin{array}{l}
u-1 \\
k-1
\end{array}\right)=\frac{1}{u}\left(\begin{array}{l}
u \\
k
\end{array}\right)
$$

we have

$$
\begin{aligned}
& \sum_{k=1}^{m-1} \frac{(-1)^{k} b_{k}(z)}{k N^{k}}+\sum_{u=2}^{m-1} \frac{(-1)^{u}}{N^{u}} \sum_{k=1}^{u-1} \frac{b_{k}(z)}{k}\left(\begin{array}{l}
u-1 \\
k-1
\end{array}\right) \\
& =\sum_{k=1}^{m-1} \frac{(-1)^{k} b_{k}(z)}{k N^{k}}+\sum_{u=2}^{m-1} \frac{(-1)^{u}}{u N^{u}} \sum_{k=1}^{u-1}\left(\begin{array}{l}
u \\
k
\end{array}\right) b_{k}(z) \\
& =-\frac{b_{1}(z)}{N}+\sum_{k=2}^{m-1} \frac{(-1)^{k}}{k N^{k}}\left(b_{k}(z)+\sum_{j=1}^{k-1}\left(\begin{array}{l}
k \\
j
\end{array}\right) b_{j}(z)\right) \\
& =\sum_{k=1}^{m-1} \frac{(-1)^{k}}{k N^{k}} \sum_{j=1}^{k}\left(\begin{array}{l}
k \\
j
\end{array}\right) b_{j}(z) .
\end{aligned}
$$


Therefore,

$$
\begin{aligned}
\gamma(z)-\gamma_{N-1}(z)= & z^{N-1}\left\{\sum_{k=2}^{m} \frac{(-1)^{k} b_{0}(z)}{k N^{k}}+\frac{(-1)^{m+1} b_{0}(z)}{(m+1) N^{m+1}} \frac{1}{\left(1+\frac{\phi_{0}}{N}\right)^{m+1}}\right. \\
& +\sum_{k=2}^{m} \frac{(-1)^{k-1} b_{k-1}(z)}{N^{k}}+\sum_{k=1}^{m-1} \frac{(-1)^{k}}{k N^{k}} \sum_{j=1}^{k}\left(\begin{array}{c}
k \\
j
\end{array}\right) b_{j}(z) \\
& +\frac{(-1)^{m}}{N^{m}} \sum_{k=1}^{m-1}\left(\begin{array}{c}
m-1 \\
k-1
\end{array}\right) \frac{b_{k}(z)}{k}+\frac{(-1)^{m+1}}{N^{m+1}} \sum_{k=1}^{m-1} \frac{b_{k}(z)}{k} \frac{\left(\begin{array}{c}
m \\
k-1
\end{array}\right)}{\left(1+\frac{\phi_{k}}{N}\right)^{m+1}} \\
& \left.-\sum_{k=1}^{m-1} \frac{(-1)^{k} b_{k}(z)}{k} \frac{1}{N^{k}}+\epsilon\right\} .
\end{aligned}
$$

Because of (2.8) and (3.3) it follows that

$$
\gamma(z)-\gamma_{N-1}(z)=z^{N-1}\left\{\sum_{k=2}^{m-1} \frac{c_{k}(z)}{N^{k}}+T_{1}+T_{2}+\epsilon\right\}
$$

where

$$
\begin{aligned}
& T_{1}=\frac{(-1)^{m} b_{0}(z)}{m N^{m}}+\frac{(-1)^{m+1} b_{0}(z)}{(m+1) N^{m+1}} \frac{1}{\left(1+\frac{\phi_{0}}{N}\right)^{m+1}}, \\
& T_{2}=\frac{(-1)^{m}}{m N^{m}} \sum_{k=1}^{m-2}\left(\begin{array}{l}
m \\
k
\end{array}\right) b_{k}(z)+\frac{(-1)^{m+1}}{(m+1) N^{m+1}} \sum_{k=1}^{m-1} b_{k}(z) \frac{\left(\begin{array}{c}
m+1 \\
k
\end{array}\right)}{\left(1+\frac{\phi_{k}}{N}\right)^{m+1}} .
\end{aligned}
$$

Observe that

$$
T_{1}+T_{2}=\frac{(-1)^{m}}{N^{m}}\left\{\frac{1}{m} \sum_{k=0}^{m-2}\left(\begin{array}{c}
m \\
k
\end{array}\right) b_{k}(z)-\frac{1}{(m+1) N} \sum_{k=0}^{m-1} b_{k}(z) \frac{\left(\begin{array}{c}
m+1 \\
k
\end{array}\right)}{\left(1+\frac{\phi_{k}}{N}\right)^{m+1}}\right\}
$$

which implies that

$$
(-1)^{m}\left(T_{1}+T_{2}\right)>0 \text {, }
$$

if

$$
N \geq \frac{\frac{1}{m+1} \sum_{k=0}^{m-1}\left(\begin{array}{c}
m+1 \\
k
\end{array}\right) b_{k}(z)}{\frac{1}{m} \sum_{k=0}^{m-2}\left(\begin{array}{c}
m \\
k
\end{array}\right) b_{k}(z)}
$$

According to (2.8) and (3.3), (3.20) is equivalent to

$$
N \geq-\frac{c_{m+1}(z)}{c_{m}(z)}
$$

Thus, if $m=2 q+1$ and $N \geq-\frac{c_{m+1}(z)}{c_{m}(z)}$, then

$$
T_{1}+T_{2}<0, \quad \epsilon<0 .
$$


As a consequence, by (3.15) we have

$$
\gamma(z)-\gamma_{N-1}(z)<z^{N-1} \sum_{k=2}^{2 q} \frac{c_{k}(z)}{N^{k}} .
$$

Similarly, if $m=2 p+2$ and $N \geq-\frac{c_{m+1}(z)}{c_{m}(z)}$, then

$$
T_{1}+T_{2}>0, \quad \epsilon>0,
$$

which implies

$$
\gamma(z)-\gamma_{N-1}(z)>z^{N-1} \sum_{k=2}^{2 p+1} \frac{c_{k}(z)}{N^{k}} .
$$

This completes the proof.

The coefficients $c_{k}(z)$ in Theorem 3.1 are computed explicitly. In the following theorem, we provide an alternative approach to compute the coefficients recursively.

Theorem 3.2 The coefficients $c_{k}(z)$ in Theorem 3.1 can be determined by the following recurrence relation:

$$
\begin{aligned}
& c_{2}(z)=\frac{1}{2(1-z)}, \\
& c_{k}(z)=\frac{z}{1-z} \sum_{j=2}^{k-1}(-1)^{k-j}\left(\begin{array}{c}
k-1 \\
j-1
\end{array}\right) c_{j}(z)+\frac{(-1)^{k}}{k(1-z)}, \quad k \geq 3 .
\end{aligned}
$$

Proof From (3.3) and (2.9) it is easy to verify that

$$
c_{2}(z)=\frac{1}{2}\left[\frac{1-z}{z} b_{2}(z)-2 b_{1}(z)\right]=\frac{1}{2(1-z)} .
$$

For $k \geq 3$, by (2.7), (2.8), and (3.3), we calculate the right-hand side of (3.23):

$$
\begin{aligned}
& \frac{z}{1-z} \sum_{j=1}^{k-1}(-1)^{k-j}\left(\begin{array}{c}
k-1 \\
j-1
\end{array}\right) c_{j}(z)+\frac{(-1)^{k}}{k(1-z)} \\
& =(-1)^{k}\left\{\frac{z}{1-z} \sum_{j=1}^{k-1} \frac{1}{j}\left(\begin{array}{c}
k-1 \\
j-1
\end{array}\right)\left[\frac{1-z}{z} b_{j}(z)-j b_{j-1}(z)\right]+\frac{1}{k(1-z)}\right\} \\
& =(-1)^{k}\left\{\frac{1}{k} \sum_{j=0}^{k-1}\left(\begin{array}{c}
k \\
j
\end{array}\right) b_{j}(z)-\frac{1-z}{z} \sum_{j=1}^{k-1}\left(\begin{array}{c}
k-1 \\
j-1
\end{array}\right) b_{j-1}(z)\right\} \\
& =\frac{(-1)^{k}}{k}\left\{\frac{1-z}{z} b_{k}(z)-k b_{k-1}(z)\right\} \\
& =c_{k}(z) .
\end{aligned}
$$

Thus, the proof is complete. 
Remark 3.1 By the above recurrence relation, we obtain the first few cases of $c_{k}(z)$ :

$$
\begin{aligned}
& c_{2}(z)=\frac{1}{2(1-z)}, \quad c_{3}(z)=-\frac{2 z+1}{3(1-z)^{2}}, \\
& c_{4}(z)=\frac{3 z^{2}+8 z+1}{4(1-z)^{3}}, \quad c_{5}(z)=-\frac{4 z^{3}+33 z^{2}+22 z+1}{5(1-z)^{4}}, \\
& c_{6}(z)=\frac{5 z^{4}+104 z^{3}+198 z^{2}+52 z+1}{6(1-z)^{5}}, \\
& c_{7}(z)=-\frac{6 z^{5}+285 z^{4}+1208 z^{3}+906 z^{2}+114 z+1}{7(1-z)^{6}} .
\end{aligned}
$$

Observing the above, we find the following properties for $c_{k}(z)$.

Theorem 3.3 For $0<z<1$, the coefficients $c_{k}(z)$ in Theorem 3.1 satisfy

$$
(-1)^{k} c_{k}(z)>0, \quad k \geq 2 .
$$

In particular, if $\frac{1}{7}<z<1$, then

$$
\left|c_{k}(z)\right|<\left|c_{k+1}(z)\right|, \quad k \geq 2 .
$$

Proof By (2.8), we have

$$
c_{k}(z)=\frac{(-1)^{k}}{k} \sum_{j=0}^{k-2}\left(\begin{array}{l}
k \\
j
\end{array}\right) b_{j}(z)
$$

which implies that (3.24) is true because $b_{j}(z)>0$ for all $j \geq 0$ and $0<z<1$. By (3.26), we further have

$$
\left|c_{k+1}(z)\right|-\left|c_{k}(z)\right|=\sum_{j=0}^{k-2}\left[\frac{1}{k+1}\left(\begin{array}{c}
k+1 \\
j
\end{array}\right)-\frac{1}{k}\left(\begin{array}{c}
k \\
j
\end{array}\right)\right] b_{j}(z)+\frac{k}{2} b_{k-1}(z) .
$$

For $1 \leq j \leq k-2$,

$$
\frac{1}{k+1}\left(\begin{array}{c}
k+1 \\
j
\end{array}\right)-\frac{1}{k}\left(\begin{array}{l}
k \\
j
\end{array}\right)=\left[\frac{1}{k+1-j}-\frac{1}{k}\right]\left(\begin{array}{l}
k \\
j
\end{array}\right) \geq 0 .
$$

Thus,

$$
\left|c_{k+1}(z)\right|-\left|c_{k}(z)\right| \geq\left[\frac{1}{k+1}-\frac{1}{k}\right] b_{0}(z)+\frac{k}{2} b_{k-1}(z) .
$$

From (2.8) it follows that

$$
b_{k-1}(z)>\frac{z}{1-z} b_{0}(z) \text {. }
$$

By (3.29) and (3.30), we obtain

$$
\left|c_{k+1}(z)\right|-\left|c_{k}(z)\right|>\left[\frac{1}{k+1}-\frac{1}{k}+\frac{k z}{2(1-z)}\right] b_{0}(z) .
$$


It is easy to verify that when $1 / 7<z<1$ there holds

$$
\frac{1}{k+1}-\frac{1}{k}+\frac{k z}{2(1-z)}>0
$$

which implies that (3.25) is true.

The following theorem gives the conditions for $N$ in a simpler form than those in Theorem 3.1 .

Theorem 3.4 Let $p$ and $q$ be any positive integers. If $N \geq(2 p+2)\left(\frac{(p+1) z}{1-z}+\frac{1}{3}\right)$, then

$$
\begin{gathered}
\gamma(z)-\gamma_{N-1}(z)>z^{N-1} \sum_{k=2}^{2 p+1} \frac{c_{k}(z)}{N^{k}}, \quad 0<z<1 . \\
\text { If } N \geq(2 q+1)\left(\left(q+\frac{1}{2}\right) \frac{z}{1-z}+\frac{1}{3}\right), \text { then } \\
\gamma(z)-\gamma_{N-1}(z)<z^{N-1} \sum_{k=2}^{2 q} \frac{c_{k}(z)}{N^{k}}, \quad 0<z<1 .
\end{gathered}
$$

Proof By (2.8) we have

$$
\begin{aligned}
& \sum_{k=0}^{m-2} \frac{b_{k}(z)}{m}\left(\begin{array}{c}
m \\
k
\end{array}\right)-\frac{1}{N} \sum_{k=0}^{m-1} \frac{b_{k}(z)}{m+1}\left(\begin{array}{c}
m+1 \\
k
\end{array}\right) \\
& \quad=\sum_{k=0}^{m-2} \frac{b_{k}(z)}{m}\left(\begin{array}{c}
m \\
k
\end{array}\right)-\frac{1}{N} \sum_{k=0}^{m-2} \frac{b_{k}(z)}{m+1}\left(\begin{array}{c}
m+1 \\
k
\end{array}\right)-\frac{m}{2 N} \frac{z}{1-z} \sum_{k=0}^{m-2}\left(\begin{array}{c}
m-1 \\
k
\end{array}\right) b_{k}(z) \\
& =\frac{1}{N} \sum_{k=0}^{m-2} b_{k}(z)\left(\begin{array}{c}
m \\
k
\end{array}\right)\left[\frac{N}{m}-\frac{1}{m+1-k}-\frac{m-k}{2} \frac{z}{1-z}\right]
\end{aligned}
$$

If $N \geq m\left(\frac{1}{3}+\frac{m}{2} \frac{z}{1-z}\right)$, we have

$$
\frac{N}{m}-\frac{1}{m+1-k}-\frac{m-k}{2} \frac{z}{1-z} \geq 0
$$

for all $k=0,1, \ldots, m-2$. This implies (3.19) is true. The proof left is similar to that of Theorem 3.1.

Remark 3.2 In Theorems 3.1 and 3.4, the conditions for $N$ are sufficient but not necessary. It is possible to relax the conditions for $N$. Even we conjecture that, for some fixed $z \in(0,1)$, Theorem 3.1 is true for any positive integer $N$.

Remark 3.3 In a nutshell, according to Theorems 3.1 and 3.4, for sufficiently large $N$ and any positive integers $p$ and $q$, we have

$$
z^{N-1} \sum_{k=2}^{2 p+1} \frac{(-1)^{k}}{k N^{k}}\left[\frac{1-z}{z} b_{k}(z)-k b_{k-1}(z)\right]<\gamma(z)-\gamma_{N-1}(z)
$$




$$
<z^{N-1} \sum_{k=2}^{2 q} \frac{(-1)^{k}}{k N^{k}}\left[\frac{1-z}{z} b_{k}(z)-k b_{k-1}(z)\right] .
$$

In particular, when $z=1 / 2$, there holds

$$
\begin{aligned}
& \left(\frac{1}{2}\right)^{N-1} \sum_{k=2}^{2 p+1} \frac{(-1)^{k}}{k N^{k}}\left[b_{k}\left(\frac{1}{2}\right)-k b_{k-1}\left(\frac{1}{2}\right)\right] \\
& <\gamma\left(\frac{1}{2}\right)-\gamma_{N-1}\left(\frac{1}{2}\right) \\
& \quad<\left(\frac{1}{2}\right)^{N-1} \sum_{k=2}^{2 q} \frac{(-1)^{k}}{k N^{k}}\left[b_{k}\left(\frac{1}{2}\right)-k b_{k-1}\left(\frac{1}{2}\right)\right],
\end{aligned}
$$

which generalizes the result (1.11) due to Chen and Han [5] for large $N$. In view of (3.36), a variety of simple inequalities for $\gamma\left(\frac{1}{2}\right)-\gamma_{N-1}\left(\frac{1}{2}\right)$ are derived for sufficiently large $N$ by choosing different parameters $p$ and $q$.

$$
\begin{aligned}
& \frac{1}{2^{N-1}}\left(\frac{1}{N^{2}}-\frac{8}{3 N^{3}}\right)<\gamma\left(\frac{1}{2}\right)-\gamma_{N-1}\left(\frac{1}{2}\right)<\frac{1}{2^{N-1}} \frac{1}{N^{2}}, \quad(p=q=1), \\
& \frac{1}{2^{N-1}}\left(\frac{1}{N^{2}}-\frac{8}{3 N^{3}}+\frac{23}{2 N^{4}}-\frac{332}{5 N^{5}}\right) \\
& \quad<\gamma\left(\frac{1}{2}\right)-\gamma_{N-1}\left(\frac{1}{2}\right) \\
& \quad<\frac{1}{2^{N-1}}\left(\frac{1}{N^{2}}-\frac{8}{3 N^{3}}+\frac{23}{2 N^{4}}\right), \quad(p=q=2), \\
& \frac{1}{2^{N-1}}\left(\frac{1}{N^{2}}-\frac{8}{3 N^{3}}+\frac{23}{2 N^{4}}-\frac{332}{5 N^{5}}+\frac{479}{N^{6}}-\frac{29,024}{7 N^{7}}\right) \\
& \quad<\gamma\left(\frac{1}{2}\right)-\gamma_{N-1}\left(\frac{1}{2}\right) \\
& \quad<\frac{1}{2^{N-1}}\left(\frac{1}{N^{2}}-\frac{8}{3 N^{3}}+\frac{23}{2 N^{4}}-\frac{332}{5 N^{5}}+\frac{479}{N^{6}}\right), \quad(p=q=3) .
\end{aligned}
$$

Remark 3.4 The inequalities for $\gamma\left(\frac{1}{t}\right)-\gamma_{N-1}\left(\frac{1}{t}\right)$ were investigated by Ma and Chen [14]. In particular, some inequalities for the cases $t=3$ and $t=4$ were presented, see [14, 15, 29]. As examples, here we apply Theorem 3.1 to obtain several simple and new inequalities related to $\gamma\left(\frac{1}{3}\right)-\gamma_{N-1}\left(\frac{1}{3}\right)$ and $\gamma\left(\frac{1}{4}\right)-\gamma_{N-1}\left(\frac{1}{4}\right)$ for sufficiently large $N$.

Case $t=3$ :

$$
\begin{aligned}
& \frac{1}{3^{N-1}}\left(\frac{3}{4 N^{2}}-\frac{5}{4 N^{3}}\right)<\gamma\left(\frac{1}{3}\right)-\gamma_{N-1}\left(\frac{1}{3}\right)<\frac{1}{3^{N-1}} \frac{3}{4 N^{2}} \\
& \frac{1}{3^{N-1}}\left(\frac{3}{4 N^{2}}-\frac{5}{4 N^{3}}+\frac{27}{8 N^{4}}-\frac{123}{10 N^{5}}\right) \\
& \quad<\gamma\left(\frac{1}{3}\right)-\gamma_{N-1}\left(\frac{1}{3}\right) \\
& \quad<\frac{1}{3^{N-1}}\left(\frac{3}{4 N^{2}}-\frac{5}{4 N^{3}}+\frac{27}{8 N^{4}}\right)
\end{aligned}
$$




$$
\begin{gathered}
\frac{1}{3^{N-1}}\left(\frac{3}{4 N^{2}}-\frac{5}{4 N^{3}}+\frac{27}{8 N^{4}}-\frac{123}{10 N^{5}}+\frac{56}{N^{6}}-\frac{17,127}{56 N^{7}}\right) \\
\quad<\gamma\left(\frac{1}{3}\right)-\gamma_{N-1}\left(\frac{1}{3}\right) \\
<\frac{1}{3^{N-1}}\left(\frac{3}{4 N^{2}}-\frac{5}{4 N^{3}}+\frac{27}{8 N^{4}}-\frac{123}{10 N^{5}}+\frac{56}{N^{6}}\right) .
\end{gathered}
$$

Case $t=4$ :

$$
\begin{aligned}
& \frac{1}{4^{N-1}}\left(\frac{2}{3 N^{2}}-\frac{8}{9 N^{3}}\right)<\gamma\left(\frac{1}{4}\right)-\gamma_{N-1}\left(\frac{1}{4}\right)<\frac{1}{4^{N-1}} \frac{2}{3 N^{2}}, \\
& \frac{1}{4^{N-1}}\left(\frac{2}{3 N^{2}}-\frac{8}{9 N^{3}}+\frac{17}{9 N^{4}}-\frac{736}{135 N^{5}}\right) \\
& \quad<\gamma\left(\frac{1}{4}\right)-\gamma_{N-1}\left(\frac{1}{4}\right) \\
& \quad<\frac{1}{4{ }^{N-1}}\left(\frac{2}{3 N^{2}}-\frac{8}{9 N^{3}}+\frac{17}{9 N^{4}}\right), \\
& \frac{1}{4^{N-1}}\left(\frac{2}{3 N^{2}}-\frac{8}{9 N^{3}}+\frac{17}{9 N^{4}}-\frac{736}{135 N^{5}}+\frac{1594}{81 N^{6}}-\frac{48,296}{567 N^{7}}\right) \\
& \quad<\gamma\left(\frac{1}{4}\right)-\gamma_{N-1}\left(\frac{1}{4}\right) \\
& \quad<\frac{1}{44^{N-1}}\left(\frac{2}{3 N^{2}}-\frac{8}{9 N^{3}}+\frac{17}{9 N^{4}}-\frac{736}{135 N^{5}}+\frac{1594}{81 N^{6}}\right) .
\end{aligned}
$$

Theorem 3.5 Let $m \geq 3$ be a positive integer. For $0<z<1$, we have the following asymptotic expansion:

$$
\gamma(z)-\gamma_{N-1}(z)=z^{N-1} \sum_{k=2}^{m-1} \frac{c_{k}(z)}{N^{k}}+O\left(\frac{z^{N-1}}{N^{m}}\right), \quad N \rightarrow \infty,
$$

where $c_{k}(z)$ are described as in (3.3).

Proof By (3.15) we immediately obtain (3.37).

Remark 3.5 When $z=1 / 2$, we recover the result of Chen and Han [5] about asymptotic expansion of $\gamma\left(\frac{1}{2}\right)-\gamma_{N-1}\left(\frac{1}{2}\right)$ :

$$
\gamma\left(\frac{1}{2}\right)-\gamma_{N-1}\left(\frac{1}{2}\right)=\frac{1}{2^{N-1}}\left(\frac{1}{N^{2}}-\frac{8}{3 N^{3}}+\frac{23}{2 N^{4}}-\frac{332}{5 N^{5}}+\frac{479}{N^{6}}-\frac{29,024}{7 N^{7}}+\cdots\right),
$$

as $N \rightarrow \infty$.

\section{Estimate of the generalized Somos' quadratic recurrence constant}

Sondow and Hadjicostas [25] generalized Somos' quadratic recurrence constant (1.3) as

$$
\sigma_{t}=\sqrt[t]{1 \sqrt[t]{2 \sqrt[t]{3 \sqrt[t]{4 \cdots}}}}=\prod_{k=1}^{\infty} k \frac{1}{k^{k}}
$$


Then they established the relation between the generalized Somos' quadratic recurrence constant $\sigma_{t}$ and the function $\gamma\left(\frac{1}{t}\right)$ :

$$
\gamma\left(\frac{1}{t}\right)=t \ln \frac{t}{(t-1) \sigma_{t}^{t-1}}, \quad t>1
$$

Recently, Coffey [6] obtained the following integral and series representations for $\ln \sigma_{t}$ :

$$
\ln \sigma_{t}=\int_{0}^{\infty}\left(\frac{e^{-x}}{t-1}+\frac{1}{1-t e^{x}}\right) \frac{d x}{x}
$$

and

$$
\ln \sigma_{t}=\frac{1}{t-1} \sum_{k=1}^{\infty} \frac{(-1)^{k-1}}{k} L i_{k}\left(\frac{1}{t}\right)=\frac{1}{t-1} \sum_{k=1}^{\infty} \frac{1}{k}\left[L i_{k}\left(\frac{1}{t}\right)-1\right]
$$

in terms of the polylogarithm function. Using (4.2) and Theorem 3.1, it is natural that we give an estimate for the generalized Somos' quadratic recurrence constant $\sigma_{t}$ :

$$
L_{t}<\sigma_{t}<U_{t}
$$

where the bounds

$$
\begin{aligned}
& L_{t}=\left\{\frac{t}{t-1} \exp \left\{-\frac{\gamma_{N-1}\left(\frac{1}{t}\right)}{t}-\frac{1}{t^{N}} \sum_{k=2}^{2 q} \frac{c_{k}\left(\frac{1}{t}\right)}{N^{k}}\right\}\right\}^{\frac{1}{t-1}}, \\
& U_{t}=\left\{\frac{t}{t-1} \exp \left\{-\frac{\gamma_{N-1}\left(\frac{1}{t}\right)}{t}-\frac{1}{t^{N}} \sum_{k=2}^{2 p+1} \frac{c_{k}\left(\frac{1}{t}\right)}{N^{k}}\right\}\right\}^{\frac{1}{t-1}} .
\end{aligned}
$$

Equivalently, (4.5) leads to a general estimate for $\ln \sigma_{t}$.

Theorem 4.1 Let $p$ and $q$ be any positive integers. For sufficiently large $N$, we have

$$
\begin{aligned}
& \frac{1}{t-1}\left\{-\frac{\gamma_{N-1}\left(\frac{1}{t}\right)}{t}-\ln \left(1-\frac{1}{t}\right)-\frac{1}{t^{N}} \sum_{k=2}^{2 q} \frac{c_{k}\left(\frac{1}{t}\right)}{N^{k}}\right\} \\
& <\ln \sigma_{t}<\frac{1}{t-1}\left\{-\frac{\gamma_{N-1}\left(\frac{1}{t}\right)}{t}-\ln \left(1-\frac{1}{t}\right)-\frac{1}{t^{N}} \sum_{k=2}^{2 p+1} \frac{c_{k}\left(\frac{1}{t}\right)}{N^{k}}\right\},
\end{aligned}
$$

where $c_{k}(z)$ are described as in (3.3).

As a consequence, we easily obtain an asymptotic expansion for $\ln \sigma_{t}$.

Theorem 4.2 As $N \rightarrow \infty$, the following asymptotic expansion holds:

$$
\ln \sigma_{t}=\frac{1}{t-1}\left\{-\frac{\gamma_{N-1}\left(\frac{1}{t}\right)}{t}-\ln \left(1-\frac{1}{t}\right)-\frac{1}{t^{N}} \sum_{k=2}^{m-1} \frac{c_{k}\left(\frac{1}{t}\right)}{N^{k}}\right\}+O\left(\frac{1}{N^{m} t^{N}}\right),
$$

where $m \geq 3$. 
Remark 4.1 Theorems 4.1 and 4.2 show that the function

$$
\frac{1}{t-1}\left\{-\frac{\gamma_{N-1}\left(\frac{1}{t}\right)}{t}-\ln \left(1-\frac{1}{t}\right)-\frac{1}{t^{N}} \sum_{k=2}^{m-1} \frac{c_{k}\left(\frac{1}{t}\right)}{N^{k}}\right\}
$$

is a good approximation of $\ln \sigma_{t}$ with the error term $O\left(1 /\left(N^{m} t^{N}\right)\right)$. In particular, for large $t$, the approximation effect is great.

When $t=2$, we have the following estimate and asymptotic expansion for $\ln \sigma$.

Corollary 4.1 Let $p$ and $q$ be any positive integers. For sufficiently large $N$, we have

$$
-\frac{\gamma_{N-1}\left(\frac{1}{2}\right)}{2}+\ln 2-\frac{1}{2^{N}} \sum_{k=2}^{2 q} \frac{c_{k}\left(\frac{1}{2}\right)}{N^{k}}<\ln \sigma<-\frac{\gamma_{N-1}\left(\frac{1}{2}\right)}{2}+\ln 2-\frac{1}{2^{N}} \sum_{k=2}^{2 p+1} \frac{c_{k}\left(\frac{1}{2}\right)}{N^{k}}
$$

Corollary 4.2 As $N \rightarrow \infty$, the following asymptotic expansion holds:

$$
\ln \sigma=-\frac{\gamma_{N-1}\left(\frac{1}{2}\right)}{2}+\ln 2-\frac{1}{2^{N}} \sum_{k=2}^{m-1} \frac{c_{k}\left(\frac{1}{2}\right)}{N^{k}}+O\left(\frac{1}{N^{m} 2^{N}}\right)
$$

where $m \geq 3$.

In fact, according to Definition (4.1) and Lemma 2.1 or (2.12), we find an alternative approach to get the estimate for $\ln \sigma_{t}$.

Theorem 4.3 Let $N$ be any positive integer. Then, for $t>1$ and $m \geq 1$, we have

$$
\ln \sigma_{t}=\lambda_{N-1}\left(\frac{1}{t}\right)-\frac{\ln N}{t^{N-1}(t-1)}-\frac{1}{t^{N}}\left\{\sum_{k=1}^{m-1} \frac{(-1)^{k} b_{k}\left(\frac{1}{t}\right)}{k N^{k}}+\kappa \frac{(-1)^{m} b_{m}\left(\frac{1}{t}\right)}{m N^{m}}\right\},
$$

where $0<\kappa<1$ and $\lambda_{n}(z)=\sum_{k=1}^{n}(\ln k) z^{k}$.

Proof Taking $f(x)=\ln x$ and $z=1 / t$ in (2.12), we immediately obtain (4.12).

It should be noted that the number $N$ in Theorem 4.3 is not restricted except $N$ is a positive integer. By Theorem 4.3, it is clear that the following corollary is true.

Corollary 4.3 For $t>1$ and $m \geq 1$, we have

$$
\ln \sigma_{t}=\lambda_{N-1}\left(\frac{1}{t}\right)-\frac{\ln N}{t^{N-1}(t-1)}-\frac{1}{t^{N}} \sum_{k=1}^{m-1} \frac{(-1)^{k} b_{k}\left(\frac{1}{t}\right)}{k N^{k}}+O\left(\frac{1}{N^{m} t^{N}}\right),
$$

as $N \rightarrow \infty$.

Also, we can obtain a new type of approximation for $\ln \sigma_{t}$ similar to Theorem 3.1. 
Corollary 4.4 For any positive integers $p, q$, and $N$, there holds

$$
\begin{aligned}
& \lambda_{N-1}\left(\frac{1}{t}\right)-\frac{\ln N}{t^{N-1}(t-1)}-\frac{1}{t^{N}} \sum_{k=1}^{2 p} \frac{(-1)^{k} b_{k}\left(\frac{1}{t}\right)}{k N^{k}} \\
& <\ln \sigma_{t}<\lambda_{N-1}\left(\frac{1}{t}\right)-\frac{\ln N}{t^{N-1}(t-1)}-\frac{1}{t^{N}} \sum_{k=1}^{2 q-1} \frac{(-1)^{k} b_{k}\left(\frac{1}{t}\right)}{k N^{k}} .
\end{aligned}
$$

In particular, when $t=2$ we have

$$
\begin{aligned}
\lambda_{N-1}\left(\frac{1}{2}\right)-\frac{\ln N}{2^{N-1}}-\frac{1}{2^{N}} \sum_{k=1}^{2 p} \frac{(-1)^{k} b_{k}\left(\frac{1}{2}\right)}{k N^{k}} \\
<\ln \sigma<\lambda_{N-1}\left(\frac{1}{2}\right)-\frac{\ln N}{2^{N-1}}-\frac{1}{2^{N}} \sum_{k=1}^{2 q-1} \frac{(-1)^{k} b_{k}\left(\frac{1}{2}\right)}{k N^{k}},
\end{aligned}
$$

and

$$
\ln \sigma=\lambda_{N-1}\left(\frac{1}{2}\right)-\frac{\ln N}{2^{N-1}}-\frac{1}{2^{N}} \sum_{k=1}^{m-1} \frac{(-1)^{k} b_{k}\left(\frac{1}{2}\right)}{k N^{k}}+O\left(\frac{1}{N^{m} 2^{N}}\right),
$$

as $N \rightarrow \infty$.

Finally, we get a new estimate for $\gamma(z)$ when $0<z<1$.

Corollary 4.5 For any positive integers $p, q$, and $N$, there holds

$$
\begin{aligned}
(1-z) z^{N-2} \sum_{k=1}^{2 q-1} \frac{(-1)^{k} b_{k}(z)}{k N^{k}} & <\gamma(z)-\left(z^{N-2} \ln N-\frac{1-z}{z^{2}} \lambda_{N-1}(z)-\frac{\ln (1-z)}{z}\right) \\
& <(1-z) z^{N-2} \sum_{k=1}^{2 p} \frac{(-1)^{k} b_{k}(z)}{k N^{k}}
\end{aligned}
$$

Proof Using relation (4.2) and Corollary 4.3 and replacing $t$ by $1 / z$, we have the desired result.

In a similar manner, we obtain the following asymptotic expansion for $\gamma(z)$.

Corollary 4.6 Let $m \geq 1$ be a positive integer. For $0<z<1$, we have

$$
\begin{aligned}
\gamma(z)= & z^{N-2} \ln N-\frac{1-z}{z^{2}} \lambda_{N-1}(z)-\frac{\ln (1-z)}{z} \\
& +(1-z) z^{N-2} \sum_{k=1}^{m-1} \frac{(-1)^{k} b_{k}(z)}{k N^{k}}+O\left(\frac{z^{N-2}}{N^{m}}\right),
\end{aligned}
$$

as $N \rightarrow \infty$.

Remark 4.2 Unlike Theorem 3.1, the number $N$ in (4.17) is not restricted except $N$ is a positive integer. In other words, identity (4.17) is true for all $N \geq 1$. 
Remark 4.3 If we approximate $\gamma(z)$ by the function $z^{N-2} \ln N-\frac{1-z}{z^{2}} \lambda_{N-1}(z)-\frac{\ln (1-z)}{z}$ as $N \rightarrow$ $\infty$, then we arrive at the error bound $O\left(z^{N-2} / N\right)$. By Theorem 3.5, we conclude that it is better that we approximate $\gamma(z)$ by its partial sum $\gamma_{N-1}(z)$ because the error bound is $O\left(z^{N-1} / N^{2}\right)$

\begin{abstract}
Acknowledgements
The author sincerely appreciates the anonymous referees for their very helpful and detailed comments, which have significantly improved the presentation of this paper
\end{abstract}

\title{
Funding
}

This work was supported by the Natural Science Foundation of Zhejiang Province (Grant No. LY18A010001) and the Ningbo Natural Science Foundation (Grant No. 2017A610140).

\section{Competing interests}

The author declares that they have no competing interests.

\section{Authors' contributions}

All authors contributed equally to the writing of this paper. All authors read and approved the final manuscript.

\section{Publisher's Note}

Springer Nature remains neutral with regard to jurisdictional claims in published maps and institutional affiliations.

Received: 19 February 2019 Accepted: 9 July 2019 Published online: 17 July 2019

\section{References}

1. Abramowitz, M., Stegun, I.A.: Handbook of Mathematical Functions with Formulas, Graphs, and Mathematical Tables, 9th edn. Applied Mathematics Series, vol. 55. Nation Bureau of Standards, Dover, New York (1972)

2. Carlitz, L.: Eulerian numbers and polynomials. Math. Mag. 32, 247-260 (1959)

3. Chen, C.P.: New asymptotic expansions related to Somos' quadratic recurrence constant. C. R. Acad. Sci. Paris, Ser. I 351, 9-12 (2013)

4. Chen, C.P.: Inequalities and asymptotic expansions for the psi function and the Euler-Mascheroni constant. J. Number Theory 163, 596-607 (2016)

5. Chen, C.P., Han, X.F.: On Somos' quadratic recurrence constant. J. Number Theory 166, 31-40 (2016)

6. Coffey, M.W.: Integral representations of functions and Addison-types for mathematical constants. J. Number Theory 157, 79-98 (2015)

7. Comtet, L.: Advanced Combinatorics, the Art of Finite and Infinite Expansions. Reidel, Dordrecht (1974)

8. Finch, S.R.: Mathematical Constants. Cambridge University Press, Cambridge (2003)

9. Guillera, J., Sondow, J.: Double integrals and infinite products for some classical constants via analytic continuations of Lerch's transcendent. Ramanujan J. 40, 247-270 (2008)

10. Hirschhorn, M.D.: A note on Somos' quadratic recurrence constant. J. Number Theory 131, 2061-2063 (2011)

11. Hsu, L.C., Shiue, J.S.: On certain summation problems and generalizations of Eulerian polynomials and numbers. Discrete Math. 204, 237-247 (1999)

12. Lampret, $\vee$.: Approximation of Sondow's generalized-Euler-constant function on the interval $[-1,1]$. Ann. Univ. Ferrara 56, 65-76 (2010)

13. Lu, D., Song, Z:: Some new continued fraction estimates of the Somos' quadratic recurrence constant. J. Number Theory 155, 36-45 (2015)

14. Ma, X.S., Chen, C.P.: Inequalities and asymptotic expansions related to the generalized Somos quadratic recurrence constant. J. Inequal. Appl. 2018, 147 (2018)

15. Mortici, C.: Estimating the Somos' quadratic recurrence constant. J. Number Theory 130, 2650-2657 (2010)

16. Mortici, C.: Optimizing the rate of convergence in some new classes of sequences convergent to Euler's constant. Anal. Appl. Singapore 8, 99-107 (2010)

17. Nemes, G.: On the coefficients of an asymptotic expansion related to Somos' quadratic recurrence constant. Appl. Anal. Discrete Math. 5, 60-66 (2011)

18. Pilehrood, K.H., Pilehrood, T.H.: Arithmetical properties of some series with logarithmic coefficients. Math. Z. 255, 117-131 (2007)

19. Pilehrood, K.H., Pilehrood, T.H.: Vacca-type series for values of the generalized Euler constant constant function and its derivative. J. Integer Seq. 13, Article ID 10.7.3 (2010)

20. Ramanujan, S.: In: Hardy, G.H., Aiyar, P.V.S., Wilson, B.M. (eds.) Collected Papers of Srinivasa Ramanujan. AMS, Providence (2000)

21. Sloane, N.J.A.: Sequences A1 16603 in the on-line encyclopedia of integer sequences. http://oeis.org/A116603

22. Somos, M.: Several constants related to quadratic recurrences. Unpublished note (1999)

23. Sondow, J.: Double integrals for Euler's constant and $\ln (4 / \pi)$ and an analog of Hadjicostas's formula. Am. Math. Mon. $112,61-65(2005)$

24. Sondow, J.: New Vacca-type rational series for Euler's constant and its "alternating" analog in $\ln (4 / \pi)$. In: Additive Number Theory, pp. 331-340. Springer, New York (2010)

25. Sondow, J., Hadjicostas, P.: The generalized-Euler-constant function $\gamma(z)$ and a generalization of Somos's quadratic recurrence constant. J. Math. Anal. Appl. 332, 292-314 (2007)

26. Wang, X., Hsu, L.C.: A summation formula for power series using Eulerian fractions. Fibonacci Q. 41, 23-30 (2003) 
27. Weisstein, E.W.: Somos's quadratic recurrence constant. MathWorld-A Wolfram Web Resource, Published electronically at http://mathworld.wolfram.com/SomossQuadraticRecurrenceConstant.html

28. Xu, A., Cen, Z:: Asymptotic expansions for the psi function and the Euler-Mascheroni constant. J. Number Theory 180 360-372 (2017)

29. Yan, H., Zhang, Q., Xu, A.: A note on inequalities related to the generalized Somos quadratic recurrence constant. Submitted

30. You, X., Chen, D.R.: Improved continued fraction sequence convergent to the Somos' quadratic recurrence constant. J. Math. Anal. Appl. 436, 513-520 (2016)

Submit your manuscript to a SpringerOpen ${ }^{\odot}$ journal and benefit from:

- Convenient online submission

Rigorous peer review

Open access: articles freely available online

High visibility within the field

- Retaining the copyright to your article

Submit your next manuscript at $\boldsymbol{\triangleright}$ springeropen.com 